\title{
Improved Selected Mapping Technique for Reduction of PAPR in OFDM Systems
}

\author{
Saruti Gupta ${ }^{1}$, Dr. Ashish Goel ${ }^{2}$ \\ Department of Electronics and Comm. Engg, JIIT, Noida, India
}

\begin{abstract}
High peak to average power ratio (PAPR) is a limiting factor towards the performance of an OFDM system. Selected Mapping (SLM) is a popular peak to average power ratio (PAPR) reduction scheme used with the OFDM systems. In this technique, $U$ set of candidate sequences are generated that leads to improvement in the PAPR reduction ability of the OFDM systems. The major concern of the conventional SLM is that as the number of candidates is increased, there is a proportional rise in the inverse fast Fourier transforms (IFFT) computations of the systems. In our article we have proposed a scheme in which we increase the number of candidate sequence as $\left(U+U^{2} / 4\right)$ that leads to improved PAPR performance of the OFDM systems without any equivalent rise in the IFFT computations. It has been demonstrated that both the simulation and analytical results are well-approximated in our proposed schemes. We also estimate the threshold value of PAPR at a fixed value of complementary cumulative distribution function (CCDF) for different number of subcarriers and candidate sequences. Results demonstrate that our proposed scheme outperforms in terms of PAPR reduction ability of the OFDM signal and obtains effective PAPR threshold values with negligible loss in BER performance of the system.
\end{abstract}

Keywords-Orthogonal Frequency Division Multiplexing (OFDM); Peak to Average Power Ratio (PAPR); Selected Mapping (SLM); Complementary Cumulative Distribution Function (CCDF); PAPR threshold

\section{INTRODUCTION}

Orthogonal frequency Division Multiplexing (OFDM) is a high speed multicarrier modulation scheme that is widely used in many of the wireless application standards such as Digital Video Broadcasting (DVB) [1], IEEE 802.11a and IEEE 802.16d based Wimax broadband access [2]. The key advantages of the OFDM system are increased data rate, high spectral efficiency and resistance against multipath fading effects [3]. High peak-to-average power ratio (PAPR) of the OFDM signal is one of the main disadvantages of the OFDM system that requires large range linear amplifiers in order to limit the non-linear distortions of the OFDM signals. To overcome these problem different categories of PAPR reduction techniques have been proposed. Signal distortion techniques such as clipping [4], clipping and filtering [5] and companding [6] have been proposed. Various Signal scrambling schemes includes coding [7], selective mapping (SLM) [8], partial transmit sequence (PTS) [9], active constellation extension (ACE) [10], tone Reservation (TR) [11] and tone injection (TI) [12]. Selective Mapping (SLM) is a well-known PAPR reduction technique, where the input data blocks are multiplied with random phase vector to yield a set of $\mathrm{U}$ independent data sequences. The time-domain candidate sequences are obtained after applying the inverse fast Fourier transform (IFFT), and out of them the one with the lowest PAPR value is chosen and transmitted towards the receiver side. With increase in number of candidates the PAPR reduction ability of the OFDM signal can be improved but this leads to equivalent rise in IFFT computations. The major concern in the conventional SLM-OFDM system is to reduce the PAPR value of the OFDM signals by increasing the number of candidates without any proportional rise in the number of IFFT's. In the literature [13-17] many PAPR reduction techniques have been proposed for enhancing the number of candidate sequences in the SLM-OFDM system. These techniques aim to increase the candidate sequence of considering effects of bit redundancy and computational complexity of the SLM-OFDM system.

In [13] Shapiro-Rudin sequence set are used as phase sequence for the SLM system in order to improve PAPR performance of the OFDM signals but these may not be the best determinate phase sequence set for SLM technique. In [14] an improved SLM scheme is designed in which $U^{2}$ number of candidates are generated by using the real parts and imaginary parts of the data. In [15] GreenOFDM Algorithm is proposed to improve the PAPR reduction capability of the OFDM signals with the same number of IFFT computations as in conventional SLM-OFDM. In this scheme the number of candidates is increased by dividing the input data into two groups each containing $U / 2$ candidates and finally merging to generate $\left(U^{2} / 4\right)$ set of candidates. A variant of GreenOFDM Algorithm is suggested in [16] where the weighting factor $\alpha$ is used to generate the number of candidates. In [17] authors use the PSK modulated signal sequences, orthogonal phase factor sets and deformation parameter set to increase the number of candidate signals thereby improving the PAPR performance of the OFDM system. Another variant of GreenOFDM Algorithm is proposed in [18] where number of candidate sequences is increased to $U^{2}$, in comparison to those in GreenOFDM Algorithm.

In contrast to the previous schemes, we have addressed more combination of candidate sequence to be generated with the same IFFT computations as in conventional SLM scheme. In our proposed scheme we have increased the number of candidate sequence as $\left(U+U^{2} / 4\right)$ that leads to improved PAPR performance of the OFDM systems without any proportional rise in the IFFT computations of the system. Here, we also study the effects for different number of subcarriers and candidate sequences to estimate the threshold value of PAPR at a fixed value of CCDF. The paper is organized as follows. In Section II conventional SLM- OFDM is discussed. 
Section III gives the details about the GreenOFDM technique, Algorithm of our proposed scheme and the effects of PAPR threshold. Section IV provides both the simulation and analytical results for our proposed schemes in terms of PAPR threshold performance and BER degradations. The conclusions are provided in Section V.

\section{CONVENTIONAL SLM-OFDM}

In an OFDM system, the input data block $X=\left[X_{0}, X_{1}, \ldots \ldots . X_{N-1}\right]$ is complex modulated using M-ary quadrature phase shift keying over $N$ orthogonal sub-carriers during parallel transmissions. The time domain OFDM sequence is then generated by using $L N$ - point IFFT to the complex modulated OFDM symbols and is given as.

$$
x(n)=\frac{1}{\sqrt{N}} \sum_{k=0}^{L N-1} X(k) \exp \left(\frac{j 2 \pi n k}{L N}\right), \quad 0 \leq n \leq L N-1
$$

where $j=\sqrt{-1}$ and $L=$ Oversampling factor. For $L=4$, the PAPR results are well-approximated to the analog symbols. Larger value of $L$ leads to rise in computational complexity rather than any gain for approximation of results.

The ratio of the peak power and the mean power represents the PAPR of the OFDM signal $x(n)$ and is expressed as.

$$
P A P R=\frac{\max _{n \in[0, L N-1]}\left\{|x(n)|^{2}\right\}}{E\left\{|x(n)|^{2}\right\}}
$$

where $E\{$.$\} is the expectation operator.$

Cumulative distribution function (CCDF) [19] is used to estimate the PAPR of the OFDM signals and is expressed as the probability that the PAPR of an OFDM signal exceeds a specific threshold value of PAPR $\left(\gamma_{0}\right)$ that is given by.

$$
C C D F\left(\gamma_{0}\right)=\operatorname{Pr} o b\left\{P A P R>\gamma_{0}\right\}
$$

The semi-empirical approximation for the CCDF of the OFDM signal as given in [20] can be expressed as.

$$
C C D F_{O F D M}\left(\gamma_{0}\right) \approx 1-\left(1-e^{-\gamma_{0}}\right)^{2.8 N}
$$

Fig. 1 shows the block diagram of conventional SLMOFDM system. In this technique, $U$ candidates sequences are produced by element wise multiplication of modulated data block $X(k)$ with the random phase vector sequence $B_{u}(k)$ where $0 \leq u \leq U-1$.

The pseudo random noise sequence $B_{u}(k)$ consists of the set of phase factors $\beta_{u}(k)$ with $\left|\beta_{u}(k)\right|=1$. The candidate sequences are then presented by.

$$
X_{u}(k)=X(k) \beta_{u}(k) \quad 0 \leq u \leq U-1
$$

The time domain candidate symbol sequence generated using $L N$ - point inverse fast Fourier transform (IFFT) can be expressed as.

$x_{u}[n]=\operatorname{IFFT}\left\{X_{u}(k)\right\}$

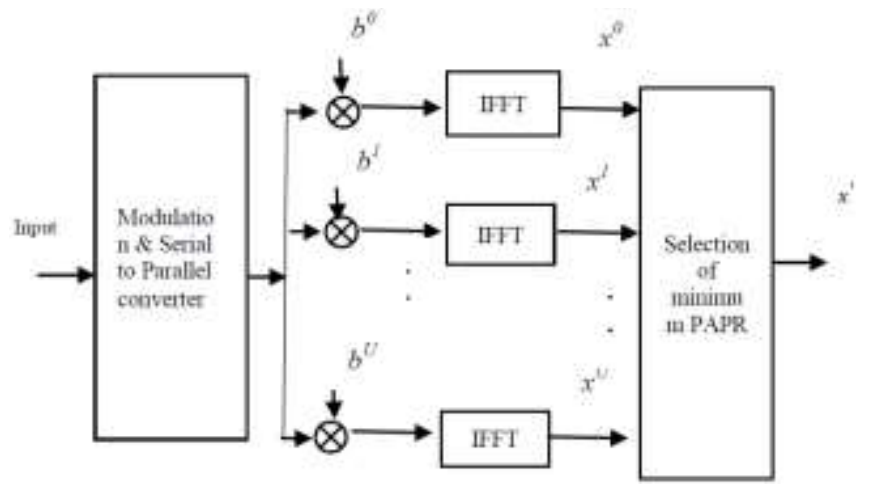

Fig. 1. Block Diagram of Conventional SLM-OFDM System.

Among these $U$ candidate sequences, the one with the minimal PAPR value is selected for transmission and satisfies the following:

$$
x^{\not t o}=\arg \min \left\{x_{u}[n]\right\}
$$

where $\mathrm{u}$ is the index of the phase set which provides the minimal PAPR value of the OFDM signal and is selected for transmission.

The semi-empirical approximation for the CCDF of the SLM-OFDM signal is analyzed as.

$$
\operatorname{CCDF}_{S L M}\left(\gamma_{0}\right) \approx\left(1-\left(1-e^{-\gamma_{0}}\right)^{2.8 N}\right)^{C_{\text {in }}}
$$

where $\mathrm{C}_{\mathrm{in}}=U$ represents the number of candidate signal generated by SLM-OFDM system.

It can be seen that the value of the CCDF decreases with increase in number of candidates in the SLM scheme but leads to rise in number of IFFT computations [21]. In order to improve the PAPR reduction capability of the OFDM signals the number of candidates in the SLM scheme should be increased in a manner that it should not affect number of IFFT's in the system. Therefore, this motivates us to design an algorithm that aims to reduce the PAPR of the OFDM signals by the increasing the number of candidates in the SLM scheme without any proportional rise in IFFT computations of the system.

\section{PROPOSED SCHEME}

\section{A. Existing Green-OFDM Algorithm}

Green-OFDM [15] was proposed to overcome the limitation of SLM-OFDM systems by increasing the number of candidates that results in improvement in the PAPR reduction ability of the OFDM signals. In this scheme the input data is split into two parts each containing $U / 2$ candidate. Here the phase vectors for the two input segments are chosen as $\left\{\beta_{g 1}(k)\right\} \in\{ \pm 1\}$ and $\left\{\beta_{g_{2}}(k)\right\} \in\{ \pm j\}$ to ensure that they do not cancel out the subcarriers of the two segments and maintain the orthogonality among the subcarriers.

These input data segments are expressed in terms of multiplication with the random phase sequences $\beta_{g l}(k)$ and $\beta_{g 2}(k)$ as. 
$X_{g 1}(k)=X(k) \beta_{g 1}(k) \quad 0 \leq g_{1} \leq \frac{U}{2}-1$

$X_{g_{2}}(k)=X(k) \beta_{g 2}(k) \quad \frac{U}{2} \leq g_{2} \leq U-1$

Then IFFT is applied to each part of the input data segments that is expressed as:

$x_{g 1}[l]=I F F T\left\{X_{g 1}(k), \beta_{g 1}(k)\right\}$

$x_{g 2}[m]=I F F T\left\{X_{g 2}(k), \beta_{g 2}(k)\right\}$

Finally these are added together to generate $\left(U^{2} / 4\right)$ candidates as given by.

$x_{g 1, g 2}[n]=x_{g 1}[l]+x_{g_{2}}[m]$

In order to preserve the Euclidean distance the sequence $\mathrm{X}_{\mathrm{g} 1, \mathrm{~g} 2}[\mathrm{n}]$ is normalized as.

$x_{g 1, g 2}[n]=\frac{x_{g 1}[l]+x_{g 2}[m]}{\sqrt{2}}$

Instead of $U$ candidates offered in conventional SLM scheme, here $\left(U^{2} / 4\right)$ set of independent candidates are generated and among these the one with the minimum PAPR value is selected for transmission.

The semi-empirical approximation for the CCDF of the Green-OFDM signal using $\mathrm{C}_{\mathrm{in}}=\left(U^{2} / 4\right)$ is then evaluated as:

$\operatorname{CCDF}_{\text {GreenSLM }}\left(\gamma_{0}\right) \approx\left(1-\left(1-e^{-\gamma_{0}}\right)^{2.8 N}\right)^{C_{\text {in }}}$

\section{B. Proposed Algorithm}

In our proposed design, a variant of GreenOFDM is designed where more possible combination of candidates are generated for a given value of $U$ candidate sequence. Here, we have utilized two segment parts, $x_{u}[n]$ consisting of set of $U$ candidates and the other segment $x_{g 1, g 2}(k)$ containing $\left(U^{2} / 4\right)$ set of candidates retaining the orthogonality between the two segment parts. The first input data segment is multiplied with $\beta_{u}(k) \in\{ \pm 1\}$ and the other data segment is rotated by $\left\{\beta_{g 1}(k)\right\} \in\{ \pm 1\}$ and $\left\{\beta_{g_{2}}(k)\right\} \in\{ \pm j\}$ ensuring the orthogonality among subcarriers for the entire segments. After applying IFFT to these data segments the time domain sequences $x_{g 1, g 2}[n]$ and $x_{u}[n]$ are finally merged to obtain $\left\{x_{u, g 1, g_{2}}[n]\right\}$ The first segment produces $U$ candidate sequence and the second group generates $U^{2} / 4$ candidate combinations.

Therefore, the number of candidate sequence equal to $\left(U+U^{2} / 4\right)$ is generated. Among these the one that satisfies the criteria of minimum PAPR that corresponds to optimal phase vector is selected for transmission. The corresponding transmitted sequence is then presented as.

$\mathrm{X}^{\prime}=\operatorname{argmin}\left\{x_{u, g 1, g 2}[n]\right\}$
The proposed scheme is described in Algorithm 1 as follows:

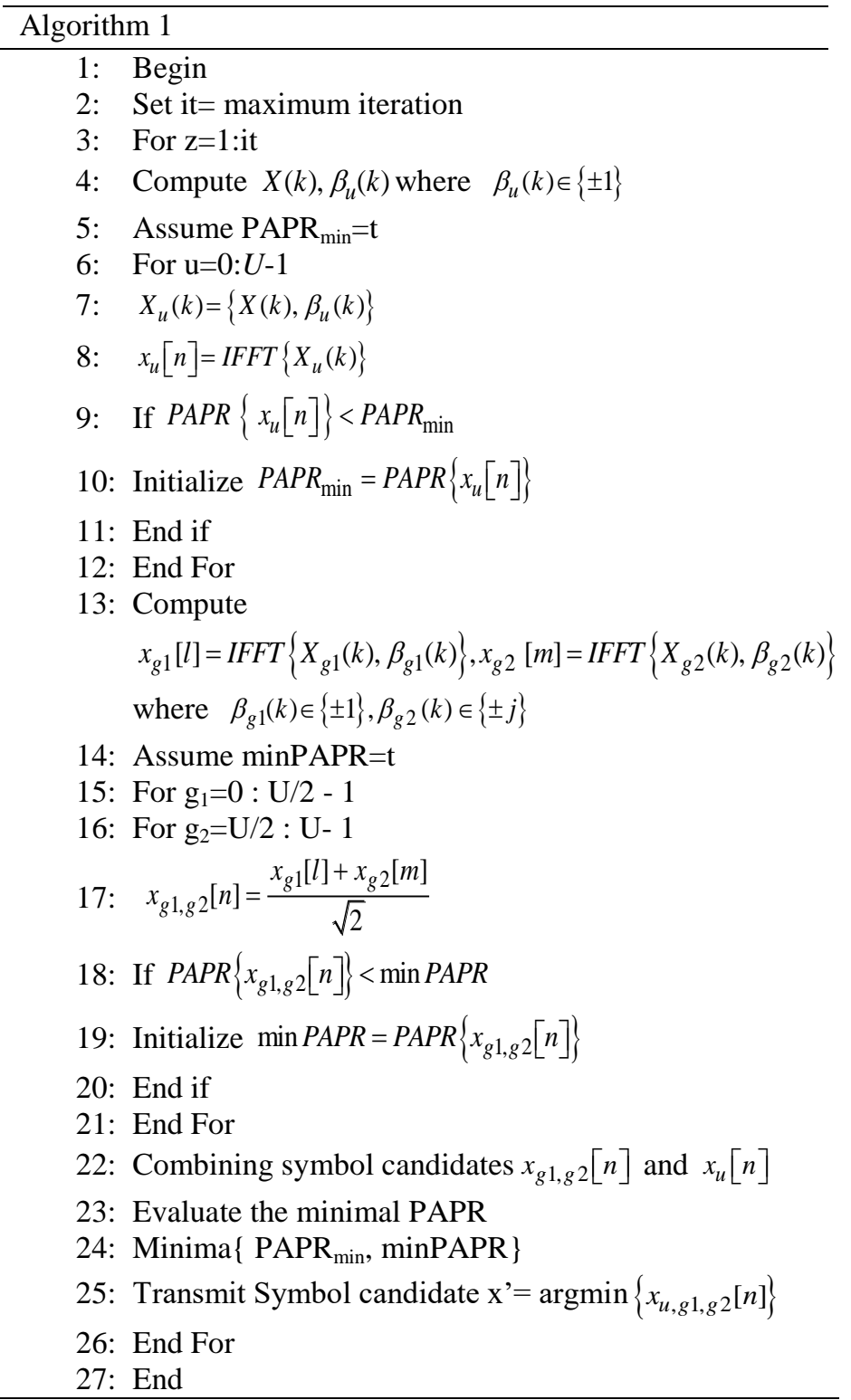

In our proposed scheme the number of candidates generated $C_{\text {in }}$ is equal to $\left(U+U^{2} / 4\right)$ with same number of IFFT computations as in conventional SLM scheme. This would result in a better CCDF value that would yield good PAPR performance of the designed system. The semi-empirical approximation for the CCDF of the proposed algorithm is then evaluated as.

$C C D F_{\text {proposed }}\left(\gamma_{0}\right) \approx\left(1-\left(1-e^{-\gamma_{0}}\right)^{2.8 N}\right)^{C_{i n}}$

\section{PAPR Threshold Parameter}

To study the effects for different values of subcarriers and candidate sequences in our proposed scheme we estimate the threshold value of PAPR $\gamma_{0}$ at a fixed value of $C C D F\left(\gamma_{0}\right)=c_{f}$. 
The expressions in (17) are rearranged to obtain the following equations of thresholds as follows:

$$
\begin{aligned}
& \gamma_{0(\text { OFDM })} \approx-\log \left(1-\left(1-c_{f}\right)^{\frac{1}{2.8 N}}\right) \\
& \gamma_{0 \text { (Proposed })} \approx-\log \left(1-\left(1-c_{f}^{1 / C_{\text {in }}}\right)^{\frac{1}{2.8 N}}\right)
\end{aligned}
$$

In the above expressions the value of number of candidates $C_{\text {in }}$ for SLM, GreenOFDM and proposed scheme is considered as $U, U^{2} / 4$ and $\left(U+U^{2} / 4\right)$. Thus, it can be noted here that the PAPR performance of the designed system at a given CCDF can also be analyzed from the number of candidates and the subcarriers.

\section{Simulation Results}

Using various computer simulations we demonstrate the PAPR performance, effects of the PAPR threshold for different values of subcarriers and sub-bands at a fixed value of $\mathrm{CCDF}=10^{-3}$, and the BER performance of our proposed scheme with the conventional SLM and the existing GreenOFDM schemes. Simulations are carried on 10000 QPSK modulated OFDM symbols for oversampling factor $L=4$, subcarriers $N=64$ and candidate sequence $U=8$. We have used AWGN channel and 3-tap Stanford University Interim (SUI) 5-multipath fading channel having average path gains [0 $\mathrm{dB},-5 \mathrm{~dB},-10 \mathrm{~dB}]$, and path delays $[0 \mu \mathrm{s}, 4 \mu \mathrm{s}, 10 \mu \mathrm{s}]$ are considered for receiver side effects under perfect side information(PSI) condition in our proposed scheme.

In Fig. 2, we have evaluated the CCDF of PAPR for our proposed scheme and compared the performance with OFDM, conventional SLM and GreenOFDM technique. Theoretical CCDF of PAPR is the upper bound of the PAPR performance and simulation results will never exceed these values. It is studied from the graph that the simulated results and analytical curves evaluated from expressions (17) are well approximated for our proposed scheme. In our proposed scheme the number of candidates $C_{i n}=U+U^{2} / 4$ results a decrease in the $C C D F\left(\gamma_{0}\right)$ value with same number of IFFT computations as required in the conventional SLM and hence improves the PAPR performance of our designed system. It is observed from the graph that at a given $\mathrm{CCDF}=10^{-3}$ the PAPR reduction ability of the proposed scheme outperforms the OFDM system by $3.9 \mathrm{~dB}$, conventional SLM by $0.6 \mathrm{~dB}$ and the GreenOFDM by $0.2 \mathrm{~dB}$.

Fig. 3 presents the PAPR thresholds $\gamma_{0}[d B]$ for different values of subcarriers $N$ ranging from 64 to 1024 at a fixed value of $C C D F\left(\gamma_{0}\right)=10^{-3}$ in our proposed scheme. The PAPR thresholds $\gamma_{0}[d B]$ are evaluated for candidate sequence $U=$ $4,8,16$ and 32 in our proposed scheme for different subcarriers.

It is seen that the value of PAPR threshold $\gamma_{0}[d B]$ increases for increasing values of subcarriers $N$. It is observed that for a fixed value of $C C D F\left(\gamma_{0}\right)=10^{-3}$, the value of $\gamma_{0}[d B]$ decreases as the candidate sequence $U$ is increased from 4 to 32 in our proposed scheme. It is studied from the graph that the simulated results and analytical curves evaluated from expressions (19) are well approximated for our proposed scheme.

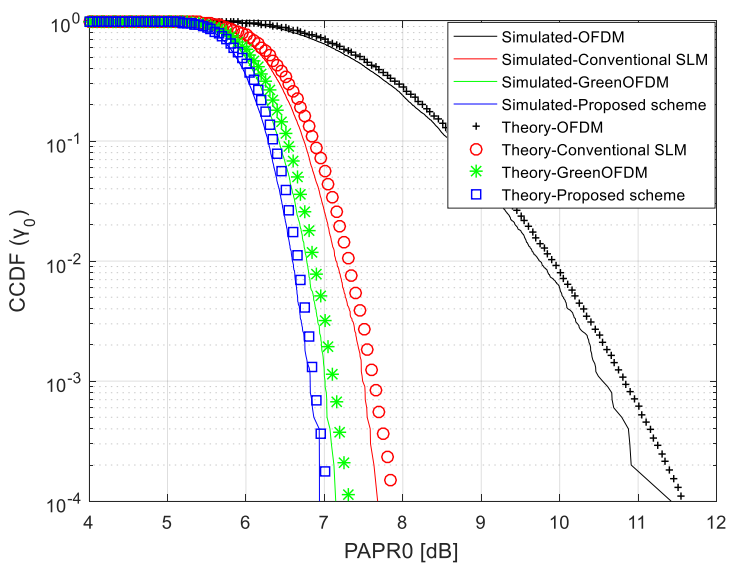

Fig. 2. PAPR Performance of Proposed Scheme and the Existing Schemes for Simulated and Approximate Results.

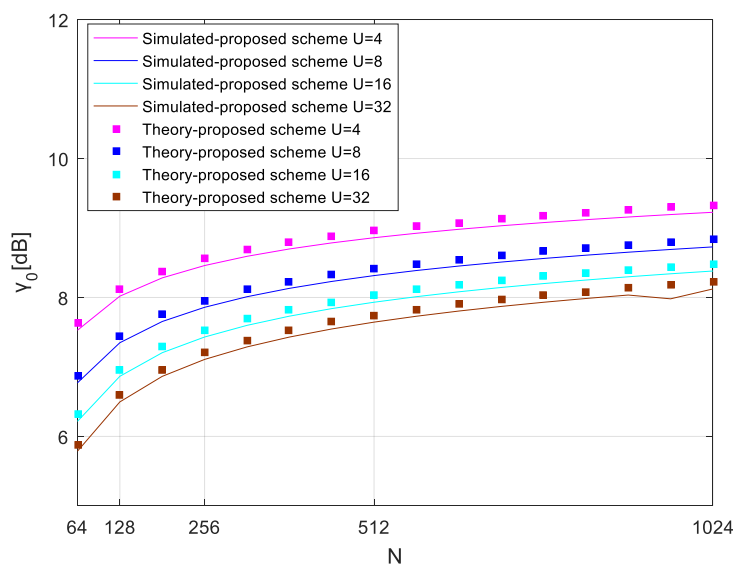

Fig. 3. $\gamma_{0}[d B]$ Versus $N$ at a Fixed $C C D F\left(\gamma_{0}\right)=10^{-3}$ for Proposed Scheme with $U=4,8,16$ and 32 with Simulated and Approximate Results.

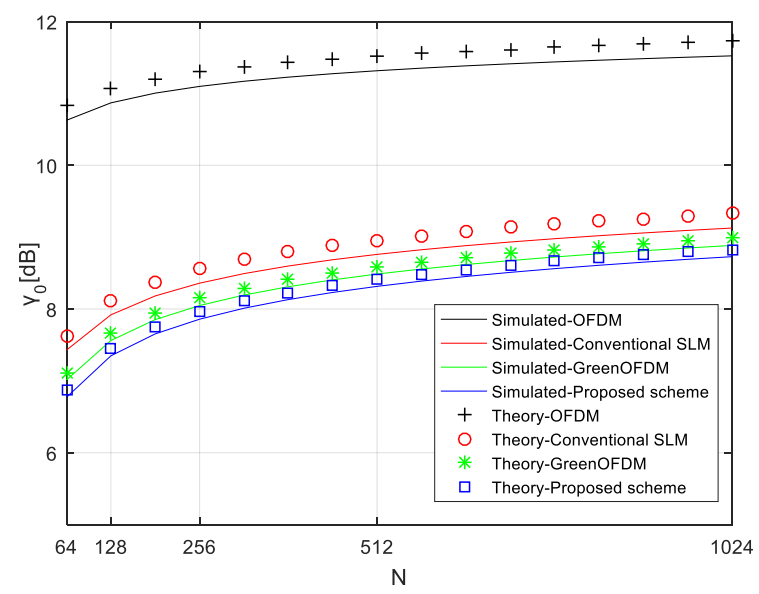

Fig. 4. $\gamma_{0}[d B]$ Versus $N$ at a Fixed $\operatorname{CCDF}\left(\gamma_{0}\right)=10^{-3}$ for Proposed Scheme and the Existing Schemes with Simulated and Approximate Results. 
In Fig. 4, we plot PAPR thresholds $\gamma_{0}[d B]$ for different number of subcarriers at a fixed value of $\operatorname{CCDF}\left(\gamma_{0}\right)=10^{-3}$ and $U=8$ for our proposed scheme and compared the performance with the OFDM, conventional SLM and GreenOFDM schemes. It is seen that the value of PAPR thresholds $\gamma_{0}[d B]$ increases for increasing values of subcarriers $N$. It is studied from these curves that the simulated results and analytical curves evaluated from expression (19) are well approximated for our proposed scheme. It is noticed that at a given $\mathrm{CCDF}=10^{-3}$ the PAPR reduction ability of the proposed scheme outperforms the OFDM system by $3.9 \mathrm{~dB}$, conventional SLM by $0.6 \mathrm{~dB}$ and the GreenOFDM by $0.2 \mathrm{~dB}$. Therefore, in our proposed scheme at a given $\operatorname{CCDF}\left(\gamma_{0}\right)=10^{-3}$ the value of $\gamma_{0}[d B]$ is lower as compared to that in OFDM, conventional SLM and GreenOFDM schemes.

Table I presents that the simulation results for different number of candidates $U$ at subcarriers $N=64$ and their comparative analysis of the PAPR performance of our proposed scheme with some of the existing schemes. It is noted that for $U=4$ the PAPR value at $\mathrm{CCDF}=10^{-3}$ for the proposed scheme, GreenOFDM [15] and conventional SLM is $7.59 \mathrm{~dB}, 8.72 \mathrm{~dB}$ and $8.35 \mathrm{~dB}$. It is found from the results that for $U=8$ at $\mathrm{CCDF}=10^{-3}$ the proposed scheme outperforms the GreenOFDM[15] and conventional SLM by $0.19 \mathrm{~dB}$ and 0.58 $\mathrm{dB}$. In the proposed scheme for $U=16$ the PAPR performance gap at $\mathrm{CCDF}=10^{-3}$ with GreenOFDM [15] and conventional SLM equals to $0.10 \mathrm{~dB}$ and $0.75 \mathrm{~dB}$.

It is also noted that for $U=32$ the PAPR value at $\mathrm{CCDF}=10^{-3}$ for the proposed scheme, GreenOFDM [15] and conventional SLM is $5.80 \mathrm{~dB}, 5.86 \mathrm{~dB}$ and $6.56 \mathrm{~dB}$. The PAPR performance gap with the existing schemes reduces for higher values of candidate set. However, the higher value of $U$ leads to more number of IFFT computations and increases the computational complexity of the system. Therefore, it is found that the proposed scheme offers a good PAPR performance with same number of IFFT computations in comparison to the existing schemes.

The BER performance of our proposed scheme, OFDM and conventional SLM over the AWGN and Rayleigh fading channel are presented in Fig. 5. It is studied from the graph that the multipath fading channel offers high BER distortions than the AWGN channel. It is noticed from the curves that under PSI condition our proposed scheme offers negligible loss in the BER performance when compared to OFDM system and the conventional SLM scheme over the AWGN and Rayleigh fading channel.

TABLE I. COMPARATIVE ANALYSIS OF THE PAPR PERFORMANCE OF PROPOSED SCHEME WITH EXISTING SCHEMES

\begin{tabular}{|l|l|l|l|}
\hline \multirow{2}{*}{$\begin{array}{l}\text { Number of } \\
\text { Candidate } \\
(\boldsymbol{U})\end{array}$} & \multicolumn{3}{|l|}{ PAPR(dB) at CCDF $\left(\mathbf{1 0}^{-3}\right)$} \\
\cline { 2 - 4 } & Conventional SLM & GreenOFDM & $\begin{array}{l}\text { Proposed } \\
\text { Scheme }\end{array}$ \\
\hline 4 & 8.35 & 8.72 & 7.59 \\
\hline 8 & 7.41 & 7.02 & 6.83 \\
\hline 16 & 6.98 & 6.33 & 6.23 \\
\hline 32 & 6.56 & 5.86 & 5.80 \\
\hline
\end{tabular}

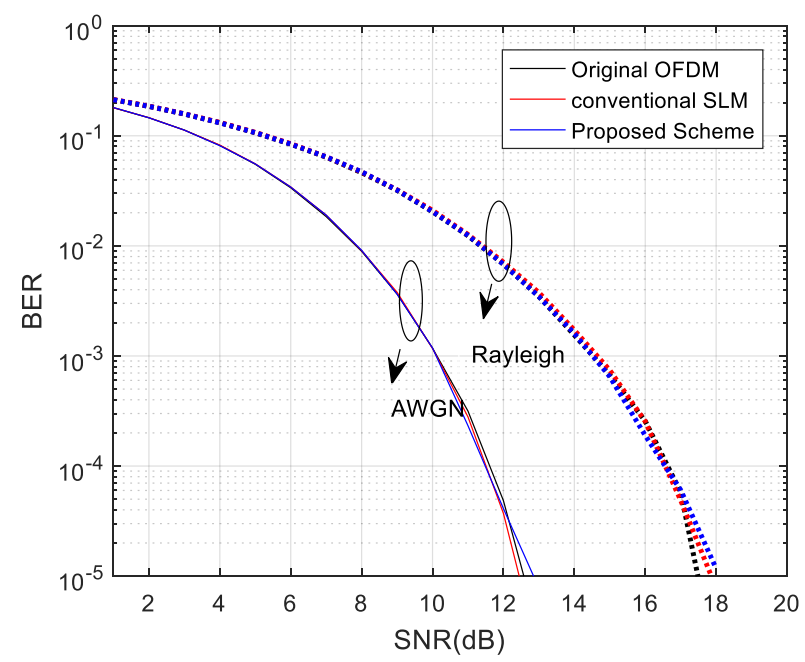

Fig. 5. BER Performance of the Proposed Scheme over AWGN and Rayleigh Fading Channels.

\section{CONCLUSION}

Our article aims to generate more set of candidate sequence that achieves effective PAPR performance of the OFDM signal without any equivalent rise in the IFFT computations. It has been demonstrated that the simulation and analytical results are well-approximated for our proposed schemes. Effects of subcarriers and candidates sequence on PAPR threshold are studied in our proposed scheme. The receiver side of our proposed scheme under PSI condition offers negligible loss in BER performance of the system as compared to OFDM system. Thus, the proposed scheme outperforms well in terms of PAPR reduction capability of the OFDM signal in comparison to that to the performance in OFDM, conventional SLM and Green-OFDM system using the same number of IFFT's.

\section{REFERENCES}

[1] Y. Wu and W. Y. Zou, "Orthogonal frequency division multiplexing: A multi-carrier modulation scheme", IEEE Trans. Consumer Electronics, vol. 41, no. 3, pp. 392-399, August 1995.

[2] W. Y. Zou and Y. Wu, "COFDM: An overview," IEEE Trans. Broadcasting, vol. 41, no. 1, pp. 1-8, Mar. 1995.

[3] T. Jiang and Y. Wu, "An overview: Peak-to-average power ratio reduction techniques for OFDM signals," IEEE Trans. Broadcast., vol. 54, no. 2,pp. 257-268, June 2008.

[4] K R Panta, J. Armstrong, “ Effects of clipping on the error performance of OFDM in frquency selective fading channel Wireless Communications" IEEE Transactions on Wireless Comm., vol. 3, no. 2, pp. 668-671, March 2004.

[5] X. Li and L. J. Cimini, Jr, "Effects of clipping and filtering on the performance of OFDM," IEEE Communications Letters, vol. 2, no. 5, pp.131-133, May 1998.

[6] T. Jiang and G. X. Zhu, "Nonlinear companding transform for reducing peak-to-average power ratio of OFDM signals," IEEE Trans. Broadcasting, vol. 50, no. 3, pp. 342-346, Sep. 2004.

[7] T. Jiang and G. Zhu, "Complement block coding for reduction in peakto-average power ratio of OFDM signals," IEEE Communications Magazine, vol. 43, no. 9, pp. S17-S22, Sep. 2005.

[8] R. W. Bauml, R. F. H. Fisher, and J. B. Huber, "Reducing the Peak-toAverage Power Ratio of Multicarrier Modulation by Selected Mapping,"IEEE Electron. Lett., vol. 32, no. 22, pp. 2056-2057, Oct.1996. 
[9] S. H. Muller and J. B. Huber, "OFDM with reduced peak-to-average power ratio by optimum combination of partial transmit sequences," IEEE Electron. Lett, vol. 33, no. 5, pp. 36-69, Feb. 1997.

[10] B.S. Krongold and D.L. Jones, "Peak Power Reduction via Active Constellation Extension," IEEE Trans. Broadcast.,vol. 49, no. 3, pp. 258-268, Sep. 2003.

[11] J. Tellado,"Multicarrier Transmission with low PAR." Ph.D.thesis, Stanford University, CA, 1998.

[12] S.H.Han, J.M.Cioffi, J.H.Lee, "Tone injection with hexagonal constellation for peak-to-average power ratio reduction in OFDM," IEEE Commun. Lett., vol. 10, no. 9, pp. 646-648, Sept. 2006.

[13] N. Ohkubo and T. Ohtsuki, "Design Criteria for Phase Sequences in Selected Mapping," IEICE Trans. Commun., vol. E86-B, no. 9, pp. 2628-2636, Sep. 2003.

[14] Fische R.F.H.:'Widely-linear selected mapping for peak-to-average power ratio reduction in OFDM', Electron. Lett., 2007,43,(14),pp.766767.

[15] J.G. Mestdagh, J.L. Gulfo Monsalve and J-M. Brossier. "GreenOFDM: a new selected mapping method for OFDM PAPR reduction". Electronics Letters, vol. 54, no. 7, pp. 449-450, 2018.
[16] D. Yu and Y. Wan, "A New Method for Reducing PAPR in OFDM System Based on GreenOFDM". 10 $0^{\text {th }}$ International Conference on Communications, Circuits and Systems (ICCCAS). pp. 227-231, Chengdu, China, 2018.

[17] Z. Zhou, L. Wang and C. Hu. "Improved SLM Scheme for Reducing the PAPR of QAM OFDM Signals". 2019 IEEE 2nd International Conference on Electronics Technology (ICET). pp.28-33, May 2019.

[18] Jorge Luis Gulfo Monsalve, Laurent Ros, Jean-Marc Brossier, Denis MestdaghAn improved GreenOFDM scheme for PAPR reduction.

[19] Wang Y, Wang LH, Ge JH, Ai B. An efficient non linear companding transform for reducing PAPR of OFDM signals IEEE Transactions on Broadcasting. 2012 Dec ;58(4):677-684.

[20] Ochiai, H., and Imai, H.: 'On the distribution of the peak-to-average power ratio in OFDM signals', Trans. Commun., 2001, 49, (2), pp. 282 289.

[21] Breiling H, Muller-Weinfurtner S H, Huber J B. SLM peak-power reduction without explicit side information[J]. IEEE Communications Letters, 2001, 5(6):239-241. 